Segarra Muñoz, L., Muñoz Vallejo, M.D. \& Segarra Muñoz, J. (2016). Empatía y educación: implicaciones del rendimiento en empatía de profesores en formación. Análisis comparativo Universidad de Castilla la Mancha y Universidad Autónoma de Chile. Revista Electrónica Interuniversitaria de Formación del Profesorado, 19(3), 173183.

DOI: http://dx.doi.org/10.6018/reifop.19.3.267331

\title{
Empatía y educación: implicaciones del rendimiento en empatía de profesores en formación. Análisis comparativo Universidad de Castilla la Mancha y Universidad Autónoma de Chile
}

Lola Segarra Muñoz ${ }^{1}$, María Dolores Muñoz Vallejo ${ }^{1}$, Juana Segarra Muñoz ${ }^{2}$

${ }^{1}$ Universidad de Castilla la Mancha, ${ }^{2}$ UNED

\section{Resumen}

Este estudio ha evaluado las habilidades empáticas de futuros profesores y las implicaciones educativas de los resultados.

Han participado 40 estudiantes de Pedagogía en Educación Física de la Facultad de Educación de Temuco (UA/Chile) y 40 estudiantes de Educación Infantil de la Facultad de Educación de Cuenca (UCLM/España).

Se ha aplicado el cuestionario TECA (2008) que incluye 4 escalas, dos destinadas a medir la empatía cognitiva: adopción de perspectivas (AP) y comprensión emocional (CE); y dos destinadas a medir la empatía afectiva: estrés empático (EE) y alegría empática (AE). Para ejercer la docencia TECA recomienda tener una empatía cognitiva alta y una empatía afectiva media o baja.

Los resultados han sido muy deficitarios en empatía cognitiva en ambas muestras (del 70\% al $85 \%$ ) y adecuados en empatía efectiva (del $85 \%$ al $98 \%$ ). El perfil global ideal solo lo consiguen el $7,5 \%$ en ambos grupos. 
Concluyendo, los maestros en formación encuestados presentan, por igual, un rendimiento muy deficitario en habilidades empáticas cognitivas, por lo que sería recomendable revisar su formación y, de forma inmediata, controlar el acceso de personal debidamente cualificado a los puestos docentes para velar por la mejora de la calidad de la enseñanza.

\title{
Palabras clave
}

Empatía; evaluación del profesor; formación de profesores.

\section{Empathy and education: trainee teachers empathy performance implications. Comparative analysis between the University of Castille la-Mancha and autonomous University of Chile}

\begin{abstract}
This study examined the empathic skills of future teachers and the educational implications of the results obtained.

40 students of Pedagogy in Physical Education at the Faculty of Education of Temuco (UA/Chile) and 40 students of Nursery and Infant Education at the Faculty of Education in Cuenca (UCLM/Spain) participated.

Students were evaluated by means of the questionnaire TECA (2008) which includes four scales, two designed to measure cognitive empathy: perspectives adoption (AP) and emotional comprehension (CE) and two designed to measure affective empathy: empathic stress (EE) and empathic happiness (AE). TECA recommends that practicing teachers should have a high level of cognitive empathy and a medium to low level of affective empathy.

The results demonstrate insufficient levels of cognitive empathy in both samples (between $70 \%$ and $85 \%$ ) and an appropriate level of affective empathy (between $85 \%$ and $98 \%$ ). The ideal global profile is achieved by only 7 ' $5 \%$ in both groups.

In summary, student teachers surveyed showed an underperformance in cognitive empathic skills. It would therefore be advisable to look at training as a matter of some urgency and control the access of suitably qualified staff to teaching posts in order to ensure an improvement in the quality of education.
\end{abstract}

\section{Key words}

Empathy; teacher education; teacher appraisal.

\section{Introducción}

Este estudio ha evaluado las habilidades empáticas de futuros profesores y las posibles implicaciones educativas de los resultados.

Numerosos autores han defendido que la Empatía es un aspecto muy importante de la inteligencia emocional que tiene múltiples aplicaciones en distintos ámbitos: 
organizacional, clínico y social (Extremera, y Fernández-Berrocal, 2004; Goleman, 1997; Salovey y Mayer, 1990; etc.)

Se trata de una importante habilidad que nos permite saber cómo se sienten las otras personas o qué están pensando, comprender las intenciones de los otros, predecir sus comportamientos y entender sus emociones. En el campo de la educación es necesario comprender las necesidades del alumno para poder ofrecer una atención de calidad (Giordani, 1997; Repeto, 1992; Rogers, 1991; Poeydomenge, 1986; etc.)

Sin embargo, no existen estudios que evalúen el rendimiento en Empatía de nuestros docentes, especialmente los que están recibiendo formación en nuestras Facultades de Educación (López-Pérez, Fernández-Pinto, Abad García, 2008; Eisenberg y Strayer, 1992; etc.)

Las fuentes teóricas de este trabajo parten de la investigación sobre empatía de LópezPérez, Fernández-Pinto, y Abad García (2008). Estos autores han defendido y demostrado que la empatía se puede medir de forma independiente. Para ello han configurado una prueba estandarizada estructurada en cuatro escalas, denominada TECA (Test de Empatía Cognitiva y Afectiva). Esta prueba es idónea para la selección de personal porque contiene perfiles diferenciados de empatía según distintas profesiones, entre ellas la docencia.

\section{Objetivos}

Se han planteado dos objetivos.

1) Medir el rendimiento en empatía actual de maestros en formación.

2) Comparar los resultados entre dos muestras de países distantes para poder analizar las posibles diferencias de perfiles empáticos.

Para ello se han analizado las respuestas de dos grupos similares de estudiantes de las Facultades de Educación de Cuenca (UCLM) y de Temuco (UA de Chile) detectando las semejanzas y diferencias entre el rendimiento en empatía cognitiva y afectiva de las dos muestras en el momento actual.

\section{Método}

Los participantes han sido, por un lado 40 estudiantes de $3^{\circ}$ curso del Grado de Pedagogía en Educación Física, de la Facultad de Educación de la Universidad Autónoma de Chile, y por otro lado 40 estudiantes de $3^{\circ}$ curso del Grado de Educación Infantil de la Facultad de Educación de Cuenca, UCLM.

La evaluación se ha realizado mediante la aplicación del cuestionario TECA (Test de Empatía Cognitiva y Afectiva, 2008) que incluye dos escalas destinadas a medir la empatía cognitiva y dos escalas relacionadas con la empatía afectiva:

Empatía cognitiva:

- Adopción de perspectivas (AP)

- Comprensión emocional (CE)

Empatía afectiva:

- Estrés empático (EE)

- Alegría empática (AE) 
La escala AP (adopción de perspectivas) hace referencia a la capacidad intelectual o imaginativa de ponerse uno mismo en el lugar de otra persona. Por nuestra experiencia, creemos que es necesario puntualizar que es una operación mental de descentración de uno mismo para comprender cómo percibe "el otro" el mundo desde su subjetividad. Por lo tanto, no existe identificación con él. Lo que ocurre es simplemente una escucha neutra para comprenderlo sin implicarse y sin dejar de ser uno mismo.

La escala CE (comprensión emocional) se refiere a la capacidad de comprender los estados emocionales, las intenciones y las impresiones de los otros. De nuevo puntualizamos que es una operación cognitiva de "comprensión" de las emociones del "otro" sin contaminación. Se entienden las emociones del otro, sin vivirlas. El empático no deja de ser él mismo durante el proceso de comunicación.

La escala EE (estrés empático) hace referencia a la capacidad para compartir las emociones negativas de las otras personas y de sintonizar emocionalmente con ellas. De nuevo puntualizamos que se trata de una forma de comunicación cognitiva en la que el otro percibe que es comprendido y que su emoción ha sido compartida cognitivamente sin llegar a reproducirla. La diferencia entre "comprender" y "sentir" radica en que el sujeto empático realiza una operación mental y no vivencia por contaminación la misma emoción que el otro. En este caso el "otro" sentirá que ha sido entendido, pero no imitado. Precisamente desde esta posición -en la que el empático comprende la emoción negativa pero no se contamina de ella- es desde donde puede surgir la ayuda. Si el empático se contaminara dejaría de ser empático para convertirse en simpático y el estado emocional de ambos sería equivalente. El posible ayudante se convertiría en otra persona necesitada de ayuda, perdiendo el control cognitivo de la situación.

La escala AE (alegría emocional) se refiere a la capacidad de comprender las emociones positivas de la otra persona. Es la vertiente positiva de la escala anterior. De nuevo puntualizamos que es una función cognitiva de comprensión del estado emocional del otro sin contaminarse. Significa comprender sus emociones, sin vivirlas.

Los niveles recomendados de empatía para ejercer la docencia, según el Test TECA, se expresa en la siguiente tabla:

Tabla 1

Perfil empático docente recomendado según el Test TECA

\begin{tabular}{|l|l|l|l|l|}
\hline Niveles & AP & CE & EE & AE \\
\hline Extremadamente alto & & & & \\
\hline Alto & $\begin{array}{l}\text { Máximo 35 } \\
\text { Mínimo 31 }\end{array}$ & $\begin{array}{c}\text { Máximo 37 } \\
\text { Mímo 32 }\end{array}$ & & \\
\hline Medio & & & Máximo 36 & Máximo 38 \\
\hline Bajo & & & Mínimo 17 & Mínimo 25 \\
\hline Extremadamente Bajo & & & & \\
\hline
\end{tabular}


Como puede apreciarse el Test TECA recomiendan los siguientes niveles para la ejercer la profesión docente con calidad:

- Empatía cognitiva: que sea alta (no extremadamente alta, media, baja o muy baja).

En AP desde una puntuación máxima de 40 puntos posibles el rango ideal del docente sería entre 31 y 35 puntos.

En CE sobre una puntuación máxima posible de 45 puntos el rango ideal del docente sería entre 32 y 37 puntos.

- Empatía afectiva: que sea media o baja (no alta, extremadamente alta, o extremadamente baja).

En EE sobre una puntuación máxima posible de 40 puntos, el rango ideal para un docente sería de 17 a 33 puntos.

En AE sobre una puntuación máxima posible de 40 puntos, el rango ideal para un docente sería entre 25 y 38 puntos.

\section{Resultados}

Una vez realizadas las mediciones en las muestras seleccionadas presentamos los resultados en dos bloques: el primero es un análisis por escalas, donde pueden observarse las puntuaciones individuales de todos los sujetos en las dos muestras; el segundo es un análisis comparativo general entre ambos grupos.

A. Por escalas

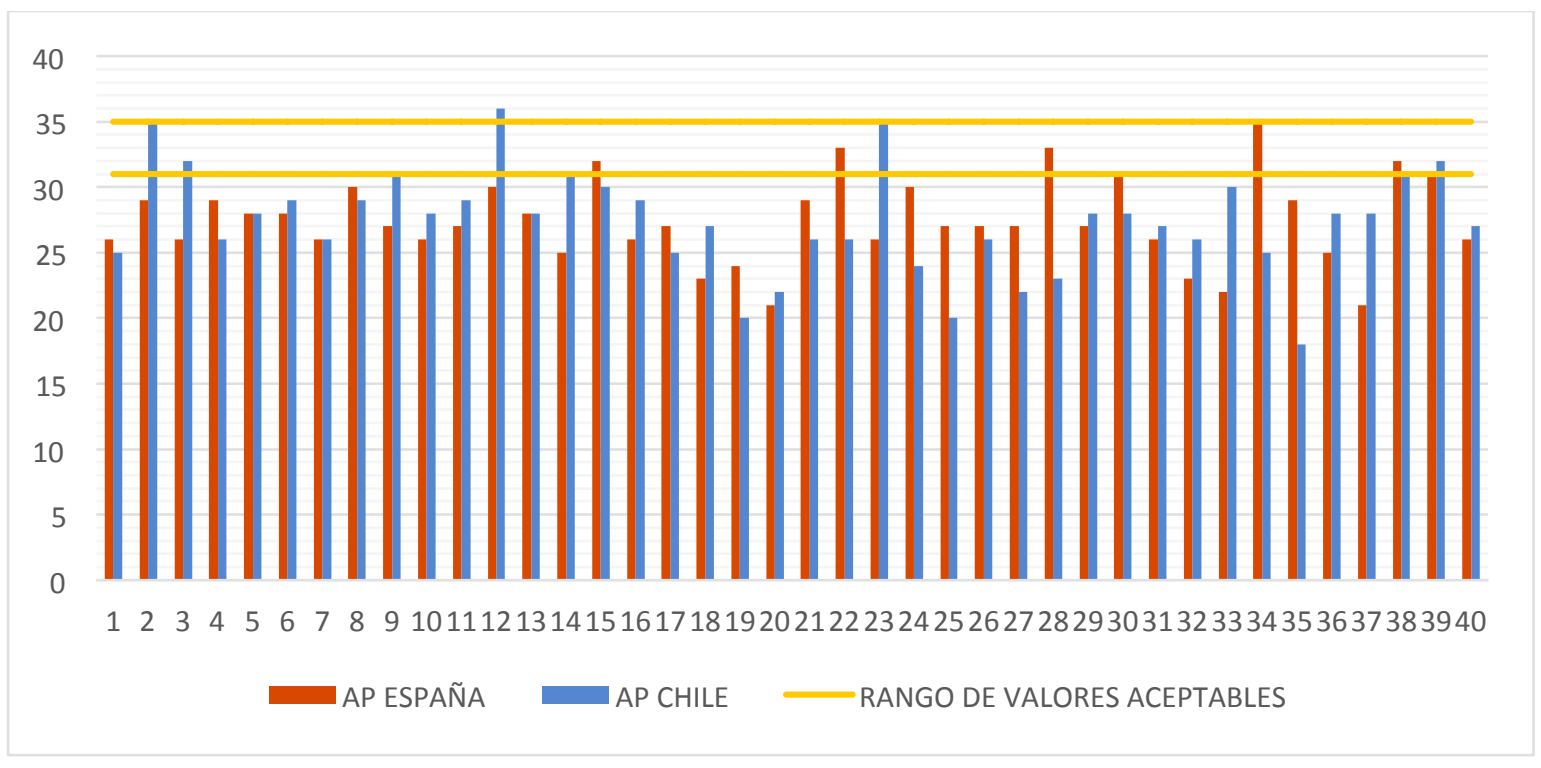

Gráfico 1. Resultados España - Chile en Adopción de Perspectivas (AP)

En empatía cognitiva según la escala de Adopción de Perspectivas (AP) los resultados muestran que solo el $15 \%$ de los alumnos españoles y el $17,5 \%$ de los chilenos se sitúan en los niveles recomendados para ejercer la profesión de docente. 
Los estudiantes restantes que son el $85 \%$ de los españoles y el $82,5 \%$ de los chilenos, están fuera de estos límites, situándose la gran mayoría extremadamente por debajo de los límites recomendados.

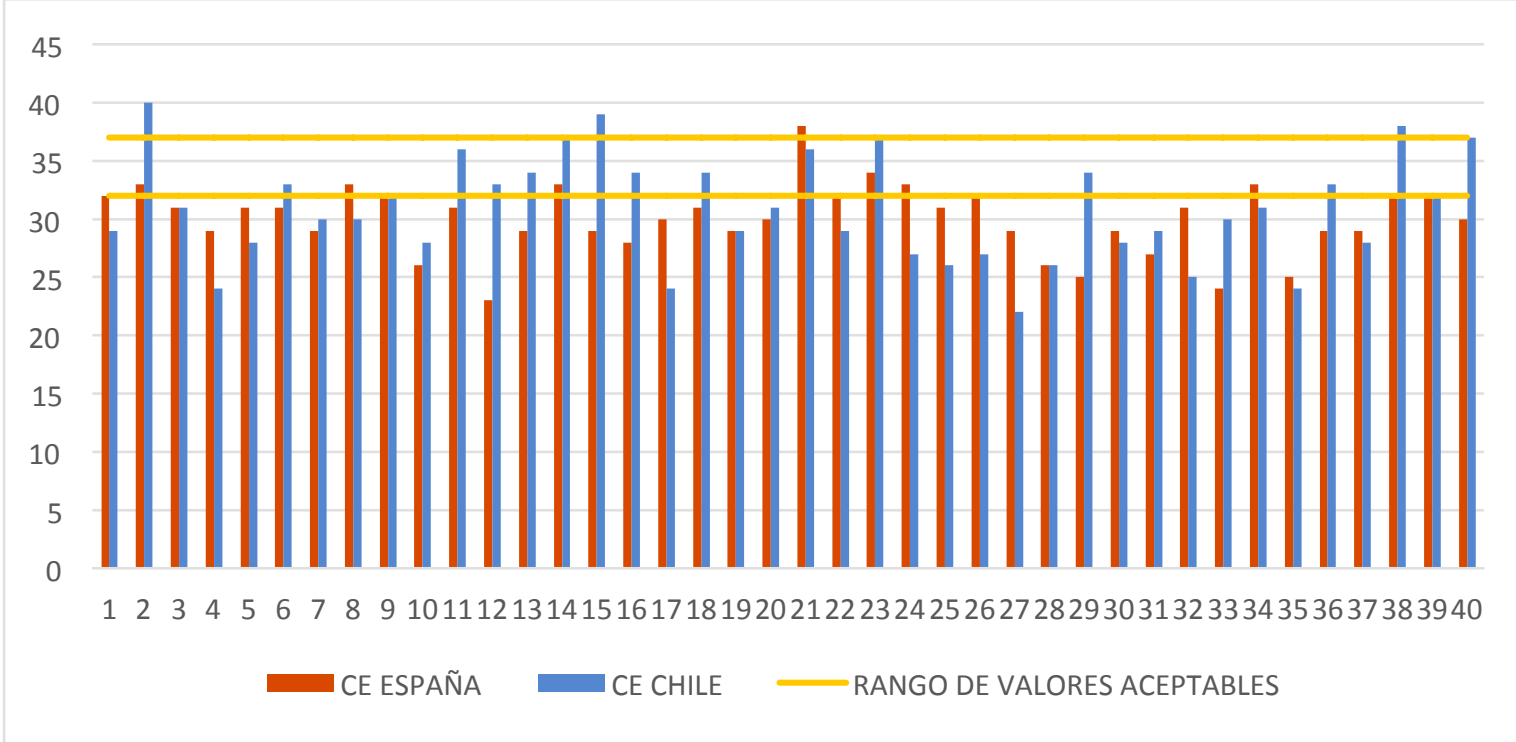

Gráfico 2. Resultados España - Chile en Comprensión Emocional (CE)

En empatía cognitiva en la escala Comprensión Emocional (CE) los resultados muestran también unas puntuaciones deficitarias ya que sólo el 30\% de los españoles y el $30 \%$ de los chilenos están en los niveles recomendados. El 70\% de los españoles y el 70\% de los chilenos están fuera del rango ideal según el TECA.

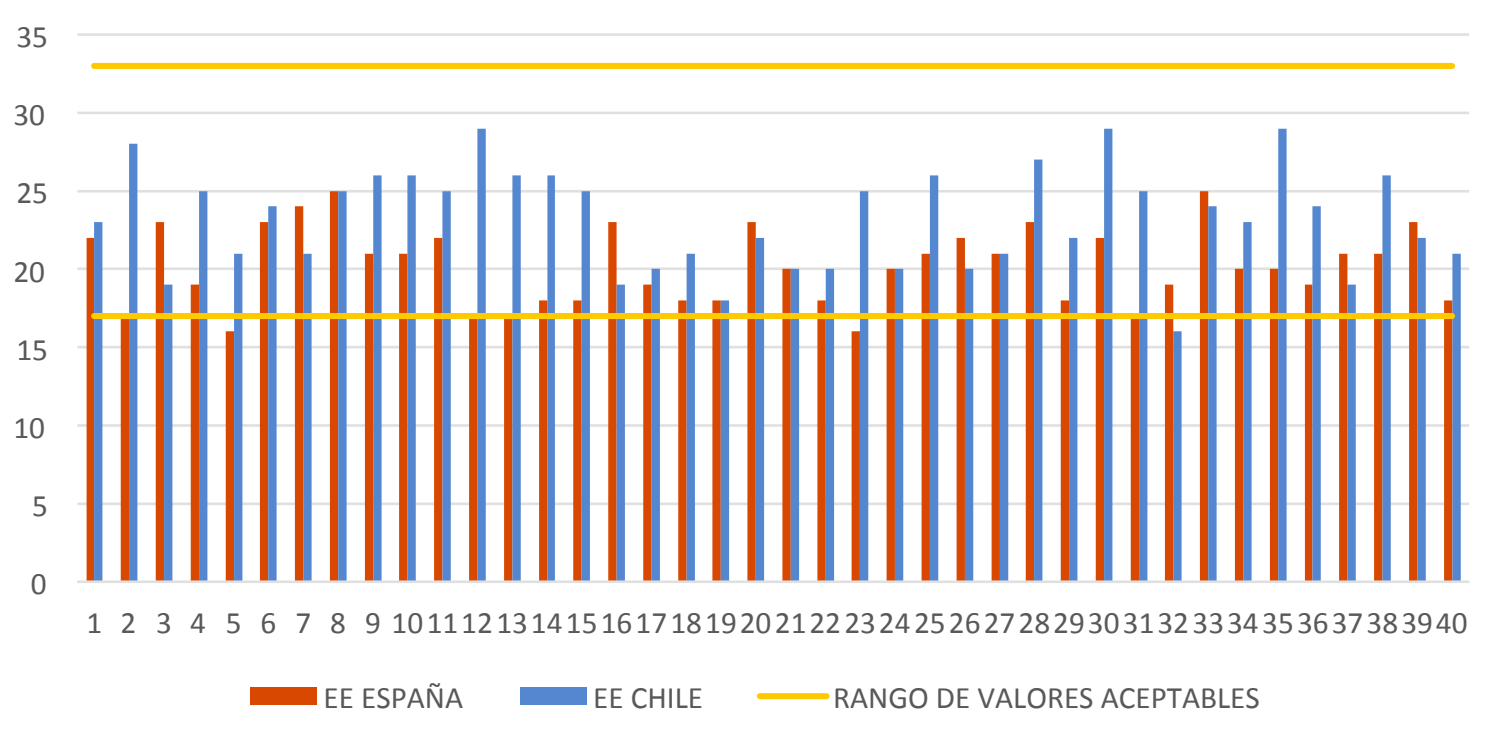

Gráfico 3. Resultados España - Chile en Estrés Empático (EE) 
En estrés empático (EE) el $95 \%$ de los estudiantes españoles se encuentran en el nivel adecuado y el $97,5 \%$ de los estudiantes chilenos, quedando un $5 \%$ de españoles y un $2,5 \%$ de chilenos fuera del umbral recomendado.

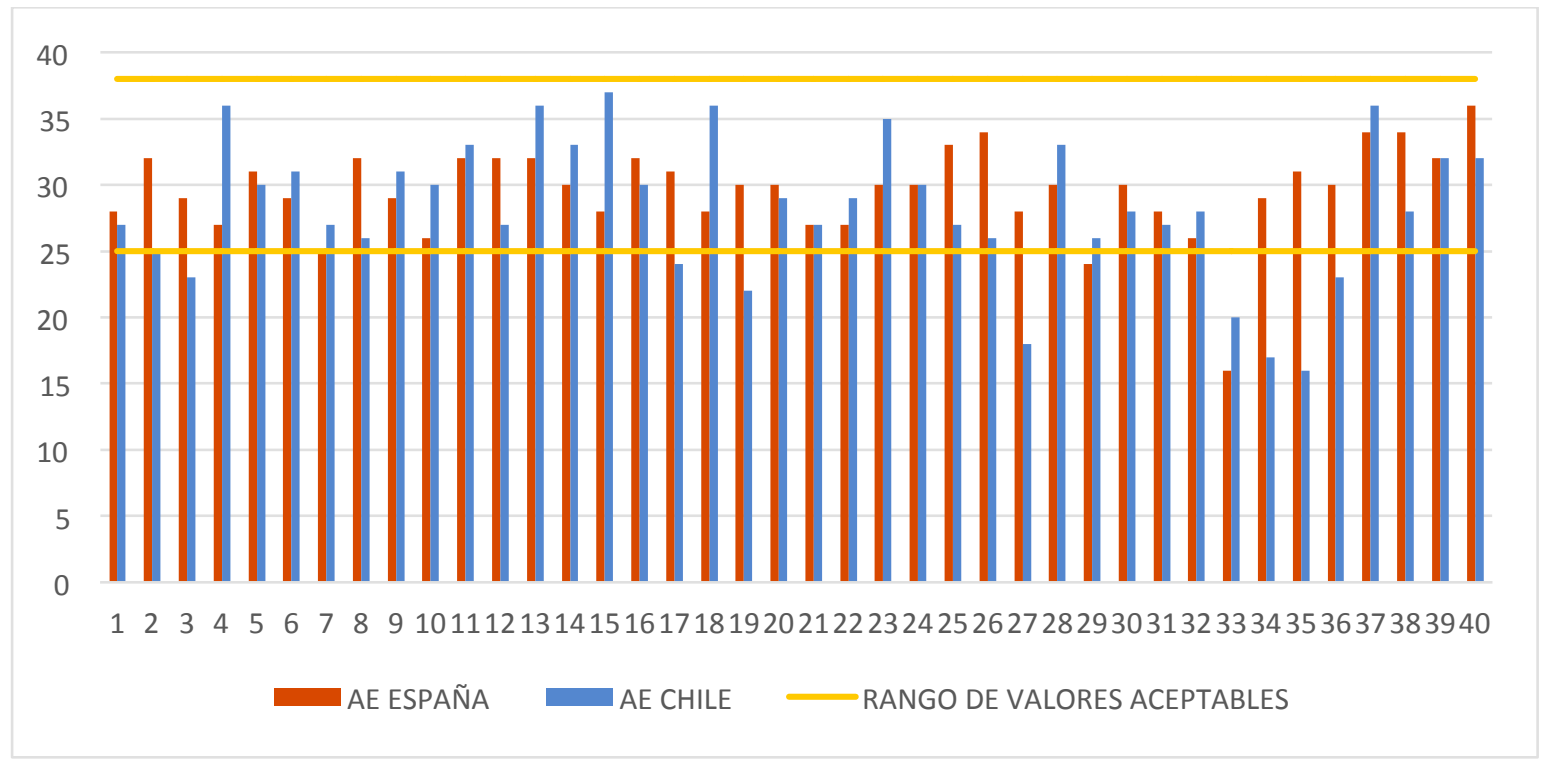

Gráfico 4. Resultados España - Chile en Alegría Empática (AE)

En la escala de Alegría Empática ( $A E$ ) el 97,5 de los españoles y el $85 \%$ de los estudiantes chilenos se encuentran dentro de los límites recomendados. Solamente el $2,5 \%$ de los españoles y el $15 \%$ de los chilenos están fuera del rango recomendado.

B. Análisis comparativo

Comparando las dos muestras de docentes en formación se obtienen los siguientes resultados:

\section{ESPAÑA \\ FACULTAD DE EDUCACIÓN CUENCA. UCLM}

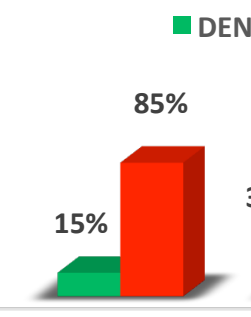

AP



CE

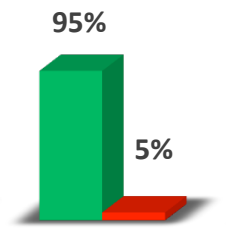

EE
$98,00 \%$



$\mathrm{AE}$

Gráfico 5. Resumen del rendimiento en empatía de maestros en formación españoles 


\section{CHILE \\ FACULTAD DE EDUCACIÓN DE TEMUCO. \\ UA}

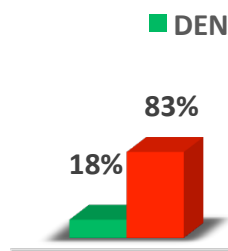

AP
DENTRO DEL LÍMITE

$70,00 \%$



CE
口 FUERA DEL LÍMITE

$98 \% \quad 85,00 \%$

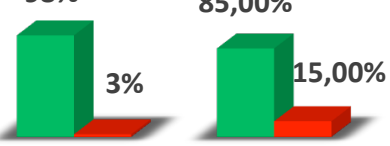

EE

$\mathrm{AE}$

Gráfico 6. Resumen del rendimiento en empatía de maestros en formación chilenos

- La mayoría de los participantes se sitúan en un nivel medio o bajo en las escalas cognitivas, cuando lo recomendable es alto.

- Los dos grupos obtienen un resultado medio o bajo en las escalas afectivas, que es el nivel recomendado.

- Los estudiantes chilenos han obtenido una pequeña ventaja en empatía cognitiva y los españoles en empatía afectiva, en relación con el perfil ideal docente.

- Todos los estudiantes están mejor capacitados para establecer relaciones de empatía afectiva que cognitiva.

\section{Discusión y Conclusiones}

Los resultados muestran que los estudiantes en formación encuestados arrojan unos datos preocupantes en habilidades sociales empáticas imprescindibles para ejercer la profesión docente con calidad, según los autores de referencia.

Ambos grupos presentan grandes déficits en habilidades cognitivas empáticas, sin embargo, presentan un nivel adecuado en habilidades empáticas afectivas.

Los porcentajes y las implicaciones docentes de los resultados serían los siguientes:

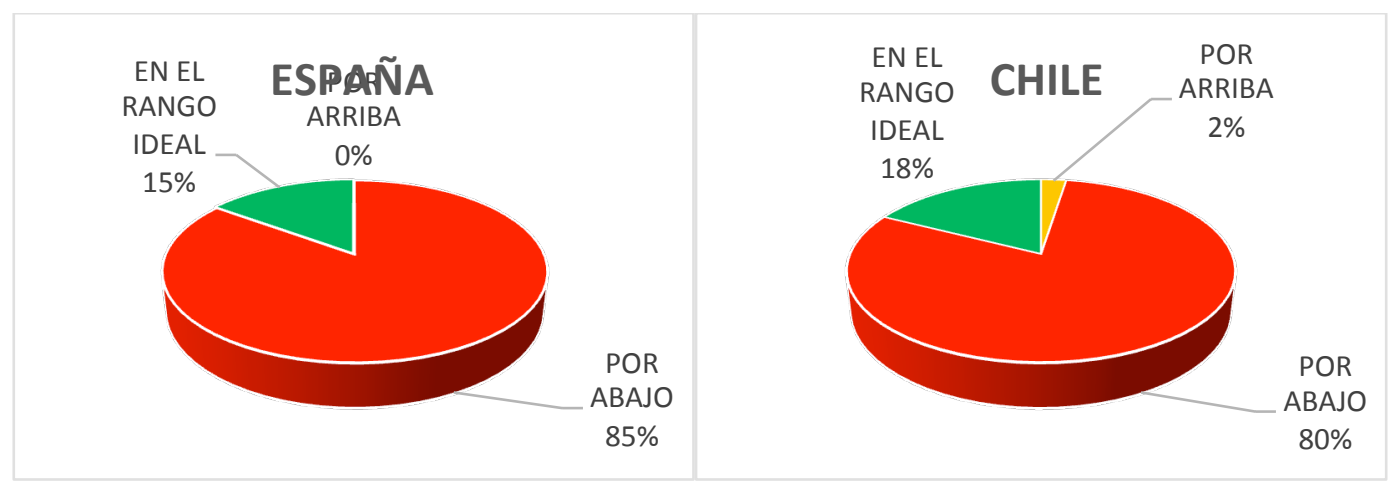

Figura 1. Implicaciones docentes España - Chile en AP 
- El 90 \% de los españoles y el $82,5 \%$ de los chilenos no tienen las competencias de "facilidad" para la comunicación, la tolerancia y la flexibilidad en las relaciones sociales.

- El gran grupo se sitúa dentro de déficits en habilidades sociales, con pensamiento rígido, y obstáculos para la comunicación con los otros.

- Únicamente en el grupo chileno existe un $2 \%$ de estudiantes que están por encima del rango recomendado.

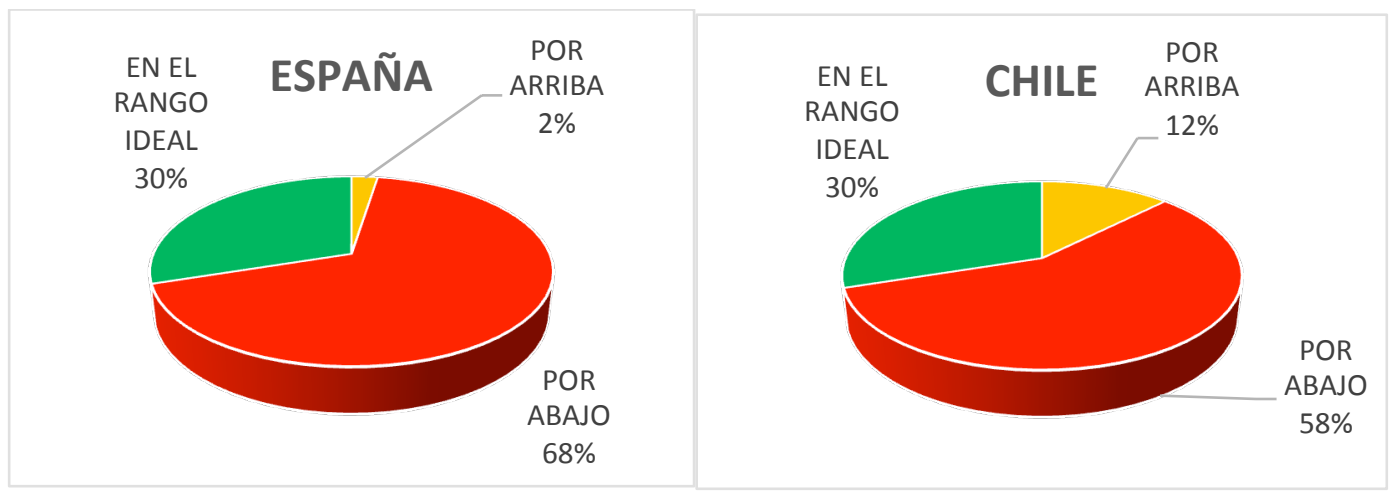

Figura 2. Implicaciones docentes España - Chile en CE

- El $68 \%$ de los estudiantes españoles y el $58 \%$ de los chilenos, tienen dificultades para reconocer y comprender los estados emocionales e intenciones de los otros.

- El $2 \%$ de los españoles y el $12 \%$ de los chilenos están por encima del rango recomendado.

- $\quad$ El 30\% de ambos se mantienen en los límites adecuados.

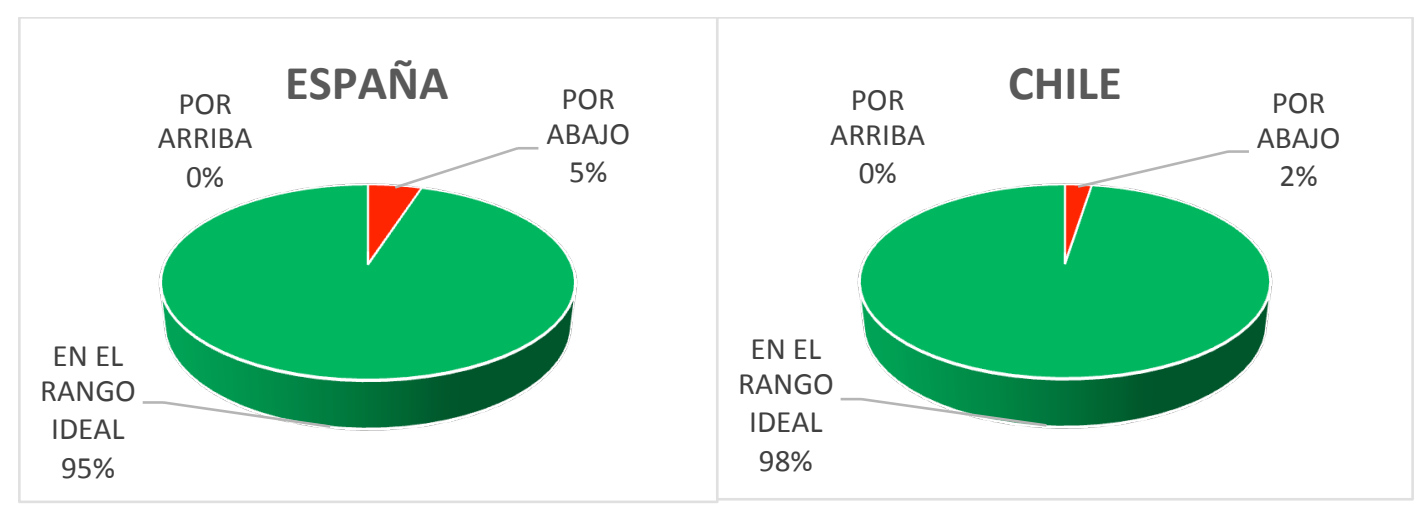

Figura 3. Implicaciones docentes España - Chile en EE

- El $5 \%$ de los estudiantes españoles y el $2,5 \%$ de los chilenos tienen dificultades para compartir las emociones negativas de los otros.

- El 95\% de los españoles 97,5\% de los chilenos están en los límites adecuados 


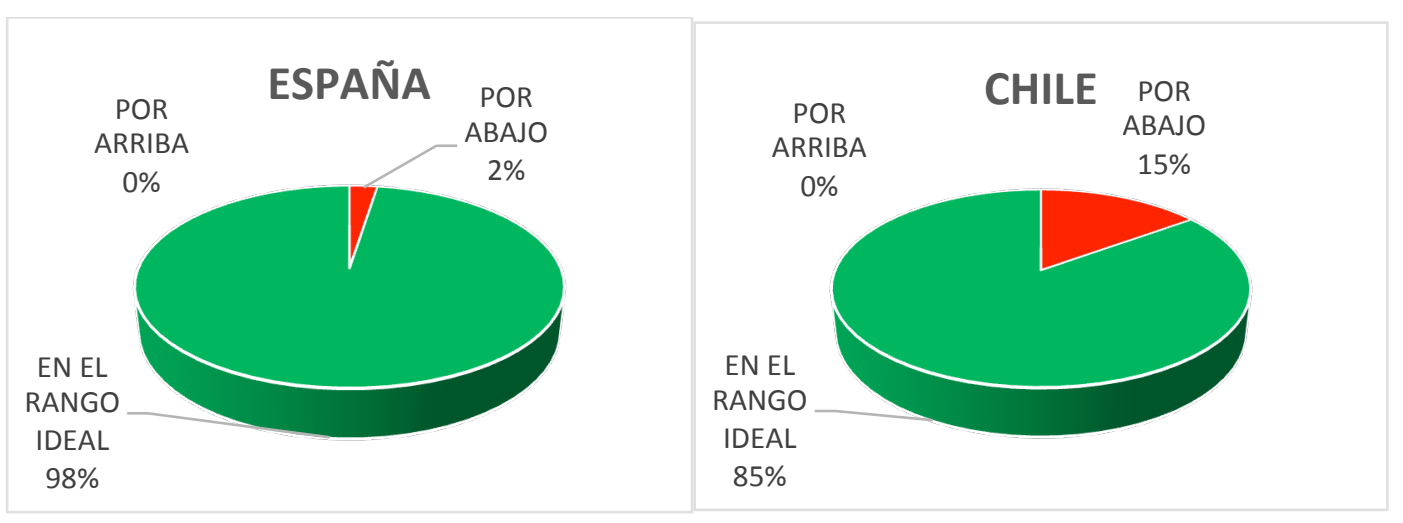

Figura 4. Implicaciones docentes España - Chile en AE

- El 2,5\% de los estudiantes españoles y el $15 \%$ de los chilenos tienen dificultades para compartir las emociones positivas de los otros, sin dejar de ser ellos mismos.

- El $97,5 \%$ de los españoles y el $85 \%$ de los chilenos están en los límites adecuados.

Concluyendo, las consecuencias inmediatas apuntan principalmente en dos direcciones:

1. De tipo educativo: que justificaría la conveniencia de entrenar a los futuros profesionales de la educación en habilidades sociales empáticas de forma sistemática, especialmente en habilidades cognitivas. Para conseguir este objetivo quizá habría que plantearse la revisión de los planes de estudios y la inclusión de competencias específicas en los módulos correspondientes.

2. De tipo social: que pondría en tela de juicio la necesidad de controlar el acceso de personal debidamente cualificado a los puestos de trabajo del sistema educativo. Para conseguir este objetivo quizá habría que plantearse la realización de unas pruebas de idoneidad para ejercer la docencia basadas en la demostración de competencias y no en la adquisición de conocimientos.

Si la sociedad del s. XXI demanda mayor calidad en nuestros sistemas educativos es necesario resolver este problema de forma inmediata ya que es evidente que está afectando de manera directa y negativa a la calidad de la enseñanza.

Para terminar, comentamos que hemos seguido realizando mediciones en otros grupos de docentes en formación y los resultados que hemos encontrando son similares, por lo que próximamente podremos generalizarlos con muestras mayores y más diversas.

\section{Referencias}

Eisenberg, N., y Fabes, R. A., (1990). Empathy: Conceptualization, measurement, and relation to prosocial behavior. Motivation and Emotion, 14, 131-149.

Eisenberg, N., y Strayer, J., (1992). La empatía y su desarrollo. Bilbao: Desclée de Brouwer.

Extremera, N. y Fernández-Berrocal, P. (2004). Inteligencia emocional, calidad de las relaciones interpersonales y empatía en los estudiantes universitarios. Clínica y Salud, 15 (2), 117-137.

Giordani, B. (1997). La relación de ayuda: de Rogers a Carkhuff. Bilbao: Desclée de Brouwer.

Goleman, D. (1997). Las raíces de la empatía. En Goleman, Inteligencia emocional (pp. 162184) Barcelona: Ed. Kairós. 
López-Pérez, B., Fernández-Pinto, I., Abad García, F. J. (2008) TECA. Test de Empatía Cognitiva y Afectiva. Madrid: TEA Ediciones.

Poeydomenge, M. I. (1986): La Educación según Rogers. Propuestas de la no directividad. Madrid: Narcea.

Repeto, E., (1992). Fundamentos de orientación. La empatía en el proceso orientador. Madrid: Ed. Morata.

Rogers, C. (1991). Libertad y Creatividad en la Educación. Barcelona: Editorial Paidós.

Salovey, O. y Mayer, J. D. (1990) Emotional Intelligence. Imagination, Cognition and Personality, 9, 185-211. 